\title{
Comments on Richards et al., Journal of Archaeological Science 35, 2008 "Strontium isotope evidence of Neanderthal mobility at the site of Lakonis, Greece using laser-ablation PIMMS”
}

\author{
G.M. Nowell ${ }^{1}$ and M.S.A. Horstwood ${ }^{2}$. \\ 1. Arthur Holmes Isotope Geology Laboratory, Northern Centre for Isotopic and Elemental \\ Tracing, Dept. of Earth Sciences, Durham University, Durham, UK DH1 3LE. \\ 2. NERC Isotope Geosciences Laboratory, British Geological Survey, Nicker Hill, Keyworth, \\ Nottingham, UK.
}

\begin{abstract}
.
We present an evaluation of the laser ablation Sr isotope data reported by Richards et al. (2008) for a Neanderthal tooth recovered from a site in Greece. Based on an alternative and analytically more robust method of correcting for isobaric interferences present during the analysis the tooth appears to be isotopically homogenous and within uncertainty of the value for modern seawater. If this is the case then contrary to the migration model proposed by Richards et al. (2008) the Neanderthal individual may have actually been a coastal dweller and lived within the vicinity of the find site.
\end{abstract}

\section{Introduction}

In the recent contribution by Richards et al (2008) Sr isotope data were presented for a single Neanderthal tooth and interpreted to represent the first 'direct evidence' for Neanderthal migration of at least $20 \mathrm{~km}$. There are various issues regarding the accuracy of the published data, which cast doubt over the robustness of this interpretation. The aim of this comment is not actually to disprove the model of Richards et al (2008) but more to illustrate to the archaeological community that the accuracy of laser ablation Sr isotope data can be very sensitive to the methods used for the correction of interferences during analysis. We will illustrate that subtle differences in those correction methods can lead to diametrically opposed conclusions.

A variety of assertions are also made by Richards et al (2008) about the merits of the laser ablation analytical method relative to more 'traditional' methods, which are either incorrect and/or are oversimplified. The remainder of the comment provides a more complete discussion of these assertions and illustrates that laser ablation is not necessarily the most appropriate analytical method for the measurement of Sr isotopes on archaeologically important samples.

\section{Analytical Method:}

The most commonly quoted advantage of measuring Sr isotopes by laser ablation MultiCollector Inductively Coupled Plasma Mass Spectrometry (MC-ICPMS), referred to as PIMMS in Richards et al. (2008), is the considerably reduced sample preparation and analysis time compared 
to TIMS or solution MC-ICPMS (eg. Copeland et al., 2008). Whilst from a very simple perspective this may appear to be true it fails to take into account the most significant disadvantage of laser ablation analysis, which is the inability to separate out the matrix. The accuracy of laser ablation $\mathrm{Sr}$ isotope data can be seriously compromised by isobaric interferences generated from matrix and plasma-derived ions (see Section 2.1). Considerable time and effort is required to correct for these interferences and prove the accuracy of the data, which more than eliminates any advantages gained from the 'reduced sample preparation and analysis time'. If this effort is not made and/or insufficient data are presented to allow independent assessment of the true accuracy of the data then laser ablation MC-ICPMS is merely a very rapid method for obtaining relatively poor quality and inaccurate Sr isotope data.

We accept there are occasions where samples have sufficiently high Sr contents combined with simple matrices and large variations in Sr isotopic composition such that rigorous interference corrections may not be necessary to begin to resolve some of the isotopic variation within and between samples. In such cases laser ablation may indeed be faster than TIMS or solution-MCICPMS. However, in reality one is rarely in the fortunate position of knowing the range in $\mathrm{Sr}$ isotope composition of a sample suite prior to analysis and if laser ablation is used in the hope that the range in $\mathrm{Sr}$ isotope composition is sufficiently large then potentially important information can be lost and false negatives could be returned.

\subsection{Interference corrections:}

Within the geochemical community considerable effort has been invested in identifying the various molecular interferences that are present in the Sr mass range during laser ablation of a wide variety of materials, including tooth enamel (summarised in Vroon et al., (2008) and references within). As an illustration of the complexity of the Sr mass spectrum during laser ablation of enamel some of the potential interferences that can be present are listed in Table 1 (see also Table 2.2.2.1 Horstwood \& Nowell 2005).

These interferences affect the ${ }^{87} \mathrm{Sr} /{ }^{86} \mathrm{Sr}$ ratio directly but also indirectly through the mass bias correction, which is based on the ${ }^{86} \mathrm{Sr} /{ }^{88} \mathrm{Sr}$ ratio, and since they cannot be removed during laser ablation it is necessary to apply accurate algebraic corrections. Various protocols have been developed for correcting laser ablation Sr data (See Vroon et al., 2008 and Horstwood et al., 2008) but the common feature to all these approaches is the recognition that the majority of the interferences are matrix sensitive and cannot be assumed to be constant during and/or between analyses. Where possible, corrections must be applied on-line in real time to each cycle/integration of an individual measurement. 
Richards et al (2008) appear to have made no attempt to identify any of the isobaric interferences present during analysis of the Neanderthal tooth although they do recognise the need to correct for isobaric interferences. Given the cup configuration of the mass spectrometer used by Richards et al (2008; Table 1) it would actually not be possible to correct for interferences with the same rigour as developed within the geochemical community since key interference monitor masses are not included (Vroon et al., 2008, Horstwood et al., 2008). Instead of real time corrections, Richards et al (2008) have applied a rather simple constant offset correction to the ${ }^{87} \mathrm{Sr} /{ }^{86} \mathrm{Sr}$ ratio, based on two reference tooth samples analysed by laser ablation MC-ICPMS. Relative to TIMS and solution MC-ICPMS reference values their laser ablation ${ }^{87} \mathrm{Sr} /{ }^{86} \mathrm{Sr}$ ratio for the cow tooth was high by $0.0015(0.21 \%)$ while the sperm whale tooth was high by only $0.0005(0.07 \%)$. Richards et al (2008) simply averaged these offsets and subtracted a constant 0.001 off the ${ }^{87} \mathrm{Sr} /{ }^{86} \mathrm{Sr}$ ratio for each analysis of the Neanderthal tooth.

If this simple approach for interference corrections were to be applied to the two standard teeth themselves the cattle tooth would be over-corrected by $0.0005(0.07 \%)$ while the sperm whale tooth would be under-corrected by 0.0005 (0.07\%). Clearly this is an inherently unsatisfactory approach to interference corrections since it fails to recognise that interferences are matrix sensitive and often time dependant. This approach also introduces a minimum uncertainty level of $0.07 \%$ to all the Neanderthal data to which this offset correction is applied. Other uncertainty contributions arising from interference corrections are then additional to this.

\section{Results and discussion:}

\subsection{Internal Sr isotope variation}

Richards et al (2008) claim to observe a variation in Sr isotope composition across the Neanderthal tooth enamel identifying 3 'distinct regions of differing isotope values'. This variation is interpreted to reflect secretion of the tooth enamel whilst the Neanderthal was living in 'distinct regions'. We are unable to concur with either the observation or the interpretation for two reasons:

Firstly, given the manner in which Richards et al (2008) apply their interference corrections, any apparent $\mathrm{Sr}$ isotope variations could easily be nothing more than analytical artefact and have no archaeological significance. Applying a constant interference correction to the laser ablation $\mathrm{Sr}$ isotope data makes the critical assumption that the magnitude of the interference correction on the ${ }^{87} \mathrm{Sr} /{ }^{86} \mathrm{Sr}$ ratio is constant, both between different teeth and/or within an individual tooth (see above). This requires a 1:1 correlation between the magnitude of the interference(s) and the $\mathrm{Sr}$ content of the tooth. If this 1:1 correlation is violated a constant correction will under- or overcorrect the ${ }^{87} \mathrm{Sr} /{ }^{86} \mathrm{Sr}$ ratio and the degree of the resulting inaccuracy will correlate with the 
Sr/interference ratio. Although $\mathrm{Sr}$ substitutes for $\mathrm{Ca}$ in enamel (hydroxyapatite) it is a nonstochiometric component so its concentration can vary quite significantly, even within an individual sample. In contrast, the main molecular interferences in the Sr mass range (eg. Ca-dimmers, Caargides and $\mathrm{CaPO}$ ) encountered during laser ablation analyses are formed from stoichiometric components of the enamel whose concentrations are less variable. The net result of this is that the $\mathrm{Sr} /(\mathrm{CaP})$ ratio can vary within and between teeth (see Simonetti et al., 2008; Horstwood et al., 2008). The constant interference correction can thus yield an apparent $\mathrm{Sr}$ isotope variation, even in an isotopically homogenous tooth, due to variation in $\mathrm{Sr}$ content (and hence the Sr/interference ratio) within the tooth, which can then easily be misinterpreted as having geographical and/or migratory significance.

It is relatively straightforward to identify whether this phenomenon can explain the variation in Sr isotope composition by plotting the ${ }^{87} \mathrm{Sr} /{ }^{86} \mathrm{Sr}$ ratio against the $\mathrm{Sr}$ concentration or $\mathrm{Sr} /(\mathrm{Ca}+\mathrm{P})$ ratio of the tooth but this information is not presented by Richards et al (2008). However, it is possible to use the value of $n$, (where $\mathrm{n}$ is essentially the length of analysis), from Table 2 of Richards et al (2008) as a proxy for Sr concentration as analyses were ceased when signal intensities dropped below $1 \mathrm{~V}{ }^{88} \mathrm{Sr}$. During a laser ablation spot analysis the ${ }^{88} \mathrm{Sr}$ intensity tends to decrease as the depth of the ablation pit increases and it becomes progressively more difficult to entrain the ablated material. Under constant laser parameters ablation spots in areas of the sample characterised by lower Sr concentrations would yield lower initial ${ }^{88} \mathrm{Sr}$ beam intensities and would drop below the $1 \mathrm{~V}$ threshold of Richards et al (2008) earlier than spots in areas with high $\mathrm{Sr}$ contents, i.e. broadly speaking the Sr content should correlate with $n$.

Figure 1 shows the final corrected ${ }^{87} \mathrm{Sr} /{ }^{86} \mathrm{Sr}$ data of Richards et al (2008) for each spot on the Neanderthal tooth as a function of the length of analysis $(1 / n)$. If the tooth was isotopically homogenous and there was no correlation with the Sr content proxy it would suggest the isotope data were accurate. However, there is a good correlation $\left(r^{2}=0.786\right)$ and this could be interpreted in one of two ways. It could simply be that the tooth is isotopically heterogeneous and the isotope variation happens to be correlated with Sr concentration. However, if one accepts that interpretation one must also accept that, given the way Richards et al (2008) apply a constant interference correction and the likelihood of additional interferences being present (as shown by Simonetti et al., 2008 and Horstwood et al., 2008), there must at least be an element of 'isotope variation' that is nothing more than analytical artefact.

Secondly, irrespective of any analytical artefacts, a Sr isotopic ratio is only 'distinct' if it falls outside of uncertainty of the other data. Without reporting within-run uncertainties or even any estimate of the overall uncertainty on the ${ }^{87} \mathrm{Sr} /{ }^{86} \mathrm{Sr}$ ratio of each analysis (Richards et al., 2008, 
Table 2), it is entirely meaningless to argue that there are 3 regions with distinct Sr isotope ratios. Fortunately, it is possible to make some rough estimates of the within-run precision on the $\mathrm{Sr}$ isotope ratios for each of the spots, again using the value $n$ from Table 2 of Richards et al (2008). Assuming the average ${ }^{88} \mathrm{Sr}$ beam intensity was $1 \mathrm{~V}$ for each $8 \mathrm{~s}$ integration and the analyses lasted between 24 and 56 s gives a within-run precision, based purely on counting statistics (leaving mass bias aside), of somewhere between 0.00017 and $0.0001(2 \sigma)$. This represents a best-case estimate, since it does not and cannot account for fluctuating isobaric interferences on the Sr mass range. It is also necessary to propagate the uncertainty associated with the interference correction into the final uncertainty on the ${ }^{87} \mathrm{Sr} /{ }^{86} \mathrm{Sr}$ ratio (albeit with difficulty since it is based on only two 'standard' teeth). Since the standard cow and whale teeth were over and under-corrected for interferences by 0.0005, respectively (Section 3.2), this represents a very minimum estimate of the uncertainty on the interference correction and gives a total propagated uncertainty on each analysis of the Neanderthal tooth of between approximately 0.00053 and $0.00051(\sim 0.07 \% 2 \sigma)$. Using these more realistic minimum total uncertainties, there is no evidence whatsoever for 3 distinct $\mathrm{Sr}$ isotopic regions within the Neanderthal tooth (open diamonds, Figure 2).

\subsection{Isotopic disequilibrium between Neanderthal tooth and Lakonis find site}

Richards et al (2008) also claim the average Sr isotope composition of the Neanderthal tooth enamel is not in equilibrium with either modern seawater or the limestone of the Lakonis site from where it was recovered (Figure 2) and hence is not what would be expected of a coastal dweller. This leads them to the interpretation that the individual probably migrated into the region sometime after an age of 9-11 years.

Interestingly, the average isotopic composition of the Neanderthal and Rhino enamel samples (Richards et al., 2008; Table 2) from the same site are within uncertainty of one another $\left({ }^{87} \mathrm{Sr} /{ }^{86} \mathrm{Sr}=0.71067 \pm 0.00099\right.$ (2SD, $\mathrm{n}=9$ ) and $0.70976 \pm 0.00035$ (2SD, $\mathrm{n}=10$ ) respectively). Furthermore, both the Neanderthal and Rhino samples are isotopically more radiogenic and outside uncertainty of the assumed limestone of the Lakonis area (Fig. 3). Nonetheless, Richards et al (2008) interpret these statistically similar ${ }^{87} \mathrm{Sr} /{ }^{86} \mathrm{Sr}$ ratios in completely different ways; the Neanderthal 'migrated' whereas the Rhino was 'closer to what we would expect for the local limestone values, and therefore it is possible that the rhino probably lived mainly near to the Lakonis site'. This difference in interpretation seems incongruous. 
There is also sufficient uncertainty over the accuracy of the Sr isotope composition of the Neanderthal tooth reported by Richards et al (2008) that we believe a migration model based solely on the laser ablation isotope data is questionable.

It is clear from Figure 1 that the Sr concentration must vary within the Neanderthal tooth and this combined with the constant interference correction approach of Richards et al (2008) would result in increasingly inaccurate ${ }^{87} \mathrm{Sr} /{ }^{86} \mathrm{Sr}$ ratios as the Sr concentration decreases (as $n$ decreases). A relationship between accuracy and Sr concentration has been observed by both Horstwood et al. (2008) and Simonetti et al. (2008). Simonetti et al (2008) compared solution and laser ablation MCICPMS Sr isotope data for 37 separate archaeological human teeth of varying Sr concentrations. Figure 3, based on data taken from Simonetti et al (2008), illustrates the offset between the non interference-corrected laser ablation ${ }^{87} \mathrm{Sr} /{ }^{86} \mathrm{Sr}$ ratios and those obtained by solution mode MCICPMS as a function of the Sr concentration within the tooth. The correlation is certainly not perfect, but this is expected since the rate of production of molecular interferences is matrix dependant and time variable. Despite the uncertainty on the correlation and the fact that the Simonetti et al. (2008) data are from a different lab using different measurement protocols Figure 3 provides us with an alternative method for making an approximate estimation of the inaccuracy of the raw data for each individual spot analysis of the Neanderthal tooth according to its $\mathrm{Sr}$ concentration. Despite the caveats above this is still a far more robust approach than applying a simple constant correction but it does require the relative Sr concentration, as given by $n$, to be converted into absolute Sr concentration (ppm) since the latter are not reported by Richards et el (2008). This can be done on the basis of the known minimum beam size $\left(1 \mathrm{~V}^{88} \mathrm{Sr}\right)$, an estimate of the ion yield during laser ablation and the volume of material ablated during each analysis. The most reliable estimate of Sr concentration in the Neanderthal tooth is the minimum value since this is based the shortest analysis ( $n=3$ ), which will have had an average ${ }^{88} \mathrm{Sr}$ beam intensity closest to the ' $1 \mathrm{~V}$ minimum beam' for its duration. Our estimate of Sr concentration for $n=3$ is approximately 50-60ppm. Since the $n=7$ analyses are approximately twice the length we can assume that the $\mathrm{Sr}$ concentration is approximately double at $100-120 \mathrm{ppm}$. Based on the regression of the data of Simonetti et al. (2008) this range in Sr concentration would predict an absolute inaccuracy of the Richards et al (2008) data of between 0.0016 and 0.00386 depending on $n$ (Figure 3).

Applying a variable correction to the Neanderthal tooth data, based on our estimates of $\mathrm{Sr}$ concentration, interpolating for variable $n$, and using the regression in Figure 3 eliminates much of the apparent isotope heterogeneity within the tooth, which we believe to be an artefact of the constant correction. The variable correction also yields new estimates for the average ${ }^{87} \mathrm{Sr} /{ }^{86} \mathrm{Sr}$ ratio 
for the tooth of between $0.70933 \pm 0.00051$ (2SD) and $0.70940 \pm 0.00085$ (2SD) based on 60-100ppm or 50-120ppm ranges in Sr concentration respectively.

Both of these values are within uncertainty of the value for modern seawater (Figure 2) and only slightly higher than the assumed Sr isotope composition of the limestone at the Lakonis recovery site and would be entirely consistent with coastal residence and no migration. There is relatively little difference between our estimate of the necessary variable interference correction on the ${ }^{87} \mathrm{Sr} /{ }^{86} \mathrm{Sr}$ ratio and the constant correction of Richards et al (2008), only 0.0006 to 0.00286 , yet it results in a completely different conclusion.

In contrast to the observations of Horstwood et al. (2008) and Simonetti et al (2008) a recent study by Copeland et al (2008) apparently found little correlation between the accuracy of raw uncorrected laser ablation Sr isotope data and Sr content. This, it was argued, was inconsistent with the 'impact of any particular interfering agents' on the Sr isotope composition, although the level of interferences were never actually monitored. Nevertheless, the average difference between their laser and solution MC-ICPMS Sr isotope compositions was $0.000583 \pm 0.001876$, which is marginally less than the 0.001 correction that Richard et al (2008) applied to the Neanderthal data. If one were to apply a constant correction using the value of 0.000583 derived from Copeland et al. (2008), and assuming no correlation between accuracy and Sr content, then one would obtain a Sr isotope composition of $0.71108 \pm 0.00099$ for the Neanderthal tooth and this would apparently uphold the Neanderthal migration model of Richards et al (2008). Using the Copeland et al (2008) observations to 'validate' the accuracy of the Richards et al (2008) data or migration model is nevertheless risky for several reasons. Firstly, of the three most detailed laser ablation Sr isotope studies on teeth (Horstwood et al., 2008; Simonetti et al., 2008; Copeland et al., 2008; in order of level of detail) it is the only one that implies the absence of interferences and suggests there is no correlation between Sr content and accuracy of the ${ }^{87} \mathrm{Sr} /{ }^{86} \mathrm{Sr}$ ratio. Secondly, on closer examination of the Copeland et al (2008) data it is clear that at Sr concentrations $<100 \mathrm{ppm}$, appropriate for the Neanderthal tooth, the accuracy of the data is actually quite poor and importantly the variation in the degree of inaccuracy of the Sr isotope composition is large (0.0022 2SD). Since this variation is not correlated to Sr content it could not be corrected for and would need to be incorporated into the final uncertainty on any Sr isotope ratio. Finally, the Copeland et al (2008) study was based on modern rodent teeth and not archaeological human teeth, so its relevance to the Neanderthal study is debatable.

What we have endeavoured to illustrate here is that use of slightly different methods for assessing the potential magnitude of the necessary interference correction(s) can yield final interference-corrected Sr isotope ratios that result in conclusions diametrically opposed to one 
another. Given the lack of essential analytical details in Richards et al (2008) it is not possible to be absolutely certain of the degree of the inaccuracy on the corrected laser ablation Sr isotope data. Since the cup configuration used by Richards et al (2008) failed to include key masses for direct monitoring of molecular interferences it is unfortunate that the raw $\mathrm{Sr}$ intensity data for the Neanderthal tooth can never be reprocessed using more rigorous correction algorithms. The only way the true accuracy of the Sr isotope data in Richards et al (2008) can be unequivocally established is to simply re-analyse the Neanderthal tooth using a method free of molecular interferences. An appropriate method would be micro-drilling and analysis by TIMS or solutionmode MC-ICPMS (see Section 4).

Even if the Sr isotope data of Richards et al (2008) were subsequently proved to be accurate and consistent with their preferred model there is still a final major problem with their published migration model, which is the complete lack of supporting Sr isotope data for local geological formations, or for the isotopic composition of local bioavailable Sr,. The importance of determining the 'local' Sr isotope composition of an archaeological site before interpreting the isotopic composition of individuals from that site in terms of local versus non-local, or migrant, populations was discussed in detail by Bentley et al (2004) but appears to have ignored in the Neanderthal study. Without local Sr isotope compositions there is no framework in which to interpret the Neanderthal tooth results.

Richards et al (2008) nevertheless assert that the ${ }^{87} \mathrm{Sr} /{ }^{86} \mathrm{Sr}$ ratios measured in the tooth are consistent with the individual living in a region with 'radiogenic, likely volcanic rocks'. They identify a small area of mid-Triassic porphyritic andesites in the Krokeai region, which is within $20 \mathrm{~km}$ of the Lakonis cave and suggest that this may have been the area in which the individual resided during their childhood. The average ${ }^{87} \mathrm{Sr} /{ }^{86} \mathrm{Sr}$ ratio of the Neanderthal tooth (0.71067) reported by Richards et al. (2008) is indeed very radiogenic but it is potentially more radiogenic than would be expected for volcanics of andesitic composition. Such volcanics would normally have $\mathrm{Sr}$ isotope compositions between 0.704 and 0.709 , depending on the tectonic setting in which they are found. Although andesites with values as extreme as 0.7136 are not unknown they are extremely uncommon. Radiogenic Sr ratios as high as 0.71067 would generally be more consistent with ancient crustal basement rocks or higher silica igneous rocks such as ryholites. It is actually quite likely that the volcanics of the Krokaei region are less radiogenic than either the tooth or indeed the limestone of the Lakonis site and for this reason it is unlikely that the volcanics they refer to are of any consequence to the migration story. Although Richards et al (2008) recognise that 'a comprehensive study of the strontium isotope values of the bedrock' needs to be carried out 
to confirm the migration hypothesis this surely should have been conducted prior to publishing or alongside the Neanderthal data and migration model.

\section{Laser ablation versus 'traditional' methods (TIMS and solution MC-ICPMS) for Sr isotope measurements}

In addition to the problems with the data, Richards et al. (2008) also make various statements about the merits of laser ablation versus what they call traditional methods that are misleading and for the benefit of the archaeological community deserve more detailed discussion.

Richards et al (2008) state that early $\mathrm{Sr}$ isotope studies of tooth enamel using the 'traditional' analytical methods of TIMS or solution-mode MC-ICPMS used large amounts of sample. They also state that their 'refined' laser ablation MC-ICPMS technique consumes less material and is 'required for Sr isotope studies of rare and important samples' such as the Neanderthal tooth. It is incorrect to assert that traditional approaches for analysing Sr isotopes are more sample destructive or consume more material than laser ablation MC-ICPMS, especially when it is noted that Richards et al (2008) had a histological section of the Neanderthal tooth made prior to laser ablation, which is about as sample destructive as one can get. Although earlier TIMS and MC-ICPMS Sr isotope studies of teeth (e.g. Bentley et al. 2006; Montgomery et al. 2003) did indeed use relatively large amounts of enamel $(20 \mathrm{mg}+)$ it is important to emphasise that this was certainly not due to limitations of 'traditional' analytical methods but due to an abundance of available material and to homogenise the sample in case there were slight isotopic heterogeneity.

\subsection{Amount of material required for analysis.}

The amount of sample material required for any Sr isotope ratio measurement is a function of the concentration of Sr in the sample material and the amount of Sr required for the measurement whether it be by TIMS, solution-mode or laser ablation-mode MC-ICPMS. The amount of Sr required for the measurement itself depends on a number of factors including the yield for the chemical pre-concentration of Sr, if it is to be analysed with a 'traditional' method, the ionisation efficiency of Sr during a TIMS analysis, or the total transport and ionisation efficiency of Sr during a solution-mode or laser ablation-mode MC-ICPMS analysis. Irrespective of the chosen analytical method the amount of sample that is ultimately consumed is also dictated by the precision and accuracy that is required on the ${ }^{87} \mathrm{Sr} /{ }^{86} \mathrm{Sr}$ ratio; the better the required precision and accuracy the greater the sample consumption. 


\subsubsection{Internal Precision of analysis.}

The Sr ion yield of TIMS, defined as the total number of ions detected/atoms loaded on the filament, can be as high as $5 \%$ when using a TaF activator loading method (Birk, 1986), although 1 to 3\% is typical. Assuming an absolute maximum Sr ion yield of 5\% 1ng Sr should yield a withinrun precision on the ${ }^{87} \mathrm{Sr} /{ }^{86} \mathrm{Sr}$ ratio, based on counting statistics and not taking into account the mass bias correction, of approximately $0.0017 \%(2 \sigma)$. Even under non ideal conditions (eg. poor sample loading) and with an ion yield of only 1\% 1ng of Sr should still theoretically yield a within-run precision on the ${ }^{87} \mathrm{Sr} /{ }^{86} \mathrm{Sr}$ ratio of $<0.004 \%$. These theoretical limits to within-run precision are supported by data obtained on 0.5 to $12 \mathrm{ng}$ standards run on the Triton at Durham University over the last 7 years (Figure 4). Since the recovery of Sr during chemical pre-concentration is generally $>90 \%$ this should mean that 1 ng of Sr extracted from a sample should yield a precision on the ${ }^{87} \mathrm{Sr} /{ }^{86} \mathrm{Sr}$ ratio of $0.004 \%$ or better. For samples with a Sr concentration between 50 and 500ppm this equates to removing a total of only 0.02 to $0.002 \mathrm{mg}$ of material, assuming $100 \%$ recovery of the material, between 1 and 10 thousand times less than the 20mg stated by Richards et al (2008) as being typical of 'traditional methods'.

The Sr ion yield for a solution-mode MC-ICPMS analysis is the ratio of the total number of ions detected to total atoms consumed where the latter is controlled by the concentration of Sr in the solution and the uptake rate of the nebuliser. Most MC-ICPMS literature provide a 'sensitivity' parameter, which is simply the total signal size in volts (V) for a 1ppm solution of the analyte element for a given uptake rate and type of nebuliser. Richards et al (2008) quote a sensitivity of $60 \mathrm{~V} \mathrm{ppm}^{-1}$ for total Sr during a solution mode MC-ICPMS analysis using a PFA-50 nebuliser but fail to quote the actual uptake rate. Although a PFA-50 nebuliser should operate at an uptake rate of $50 \mu \mathrm{lmin}^{-1}$ six similar nebulisers used in Durham over 7 years all operate between 90 and $120 \mu \mathrm{l}$ $\mathrm{min}^{-1}$. A sensitivity of $60 \mathrm{~V} \mathrm{ppm}^{-1}$ at an average uptake rate of $100 \mu \mathrm{min}^{-1}$ and with $10^{11} \Omega$ resistor amplifiers gives a typical solution-mode MC-ICPMS ion yield for Sr of just $0.033 \%$. This is also very similar the ion yield for the same sample introduction set up on our Neptune instrument. With an ion yield of $0.033 \%$ a solution-mode MC-ICPMS analysis consuming a total of 1ng of Sr would provide a theoretical maximum within-run precision on the ${ }^{87} \mathrm{Sr} /{ }^{86} \mathrm{Sr}$ ratio of just less than $0.021 \%$ $(2 \sigma)$. This is approximately 12 times lower than the best precision that could be obtained by TIMS. To obtain a within-run precision of $0.002 \%$ with an ion yield of $0.033 \%$ approximately $100 \mathrm{ng}$ of $\mathrm{Sr}$ would be required compared to the $0.5-1 \mathrm{ng}$ for a TaF activator TIMS analysis. This is confirmed by data for the NIST SRM 987 Sr isotope reference material obtained on the Durham Neptune over 7 years (Figure 4). To extract 100ng of Sr from a sample with a Sr concentration between 50 and 
500ppm would require removing between 2 and $0.2 \mathrm{mg}$ of material. Solution MC-ICPMS therefore requires more material than TIMS to achieve equivalent data quality.

The Sr ion yield or sensitivity for laser ablation MC-ICPMS is rarely quoted since it can vary considerably and depends on many variables including but not restricted to the matrix being ablated, the laser ablation parameters and whether a static (single spot) or dynamic (raster) ablation was used. An estimate based on data presented in Horstwood et al (2008) suggests Sr ion yields during dynamic laser ablation can approach those of solution-mode MC-ICPMS. Richards et al (2008), used static ablation which may result in slightly lower ion yields due to the increased difficulty of entraining material into the carrier gas as the ablation pit increases in depth. Nevertheless, if it is assumed that laser ablation does give similar ion yields to solution-mode MCICPMS then theoretically it should provide a comparable level of within-run precision on similar quantities of Sr. In reality, however, the theoretical limits of within-run precision, based purely on counting statistics, are rarely attained with laser ablation. This is because compared to solutionmode MC-ICPMS analyses where the matrix is simple, laser ablation analyses are accompanied by a complex matrix and simple counting statistics fails to take into account the additional uncertainty that arises from sporadic matrix-related interferences (Section 2.1). This is clearly illustrated in Figure 4 where there is a clear difference between the theoretical and actual within-run precisions for the laser ablation analysis of an apatite from Horstwood et al (2008). In order to obtain a withinrun precision of $0.002 \%$ a laser ablation spot analysis would actually have to consume more than the 100ng of Sr that would be required for a solution-mode MC-ICPMS analysis, despite having similar ion yields (see above).

Based on the maximum within run precision for the data in Richards et al (2008; see Section 3.1) and assuming a simialar ion yield to solution-mode MC-ICPMS, the amount of Sr consumed in each analysis of the Neanderthal tooth is estimated at approximately 1-2ng as shown by the shaded box in Figure 4. Analysis of the same quantity of Sr by TIMS would have yielded an ${ }^{87} \mathrm{Sr} /{ }^{86} \mathrm{Sr}$ ratio with an order of magnitude better within-run precision (Figure 4) and with greater accuracy.

\subsubsection{Accuracy of analysis.}

Although blank contamination and mass fractionation (TIMS) or mass bias (MC-ICPMS) affect the accuracy of $\mathrm{Sr}$ isotope ratio measurements they are relatively minor compared to the effects from isobaric interferences, which vary significantly in number and intensity among the three analytical methods.

With TIMS the accuracy of the ${ }^{87} \mathrm{Sr} /{ }^{86} \mathrm{Sr}$ ratio is usually better than $50 \mathrm{ppm}$ for any beam size since the mass spectrum is essentially devoid of isobaric interferences other than Rb (assuming 
good chemical separation of Sr from the sample matrix. Table 1). With solution-mode MC-ICPMS accuracy can approach that of TIMS at larger beam intensities $\left(>5 \mathrm{~V}{ }^{88} \mathrm{Sr}\right)$, assuming good separation of $\mathrm{Sr}$ from sample matrix, but at lower beam intensities $\left(<1 \mathrm{~V}{ }^{88} \mathrm{Sr}\right)$ accuracy degrades noticeably due to the isobaric interference from the minor amounts of $\mathrm{Kr}$ usually present in the $\mathrm{Ar}$ gas (Table 1). It is precisely because of these differences in the relationship between accuracy and sample size that we would advocate analysing small samples, with 50ng or less total separated Sr, by TIMS while larger samples, in excess of 50ng, can be analysed by solution-mode MC-ICPMS.

During laser ablation MC-ICPMS accuracy is seriously compromised because of the numerous potential isobaric interferences present in the Sr mass range (Table 1; Section 2.1). There can be a strong dependence of accuracy with the composition of the matrix being ablated and with the Sr beam intensity during analysis (Figure 4 of Horstwood et al., 2008). If the Sr beam decreases due to a decrease in the concentration of $\mathrm{Sr}$ in the sample then the Sr/interferences ratio decreases and the Sr isotope ratios can become increasingly inaccurate. If, however, the Sr beam decreases due to loss of instrument sensitivity or decrease in ablation rate the Sr/interferences ratio will remain constant and the inaccuracy should also remain constant.

With laser ablation MC-ICPMS, corrections should always be applied for isobaric interferences and even then the accuracy of the Sr isotope ratios cannot be guaranteed. In contrast, TIMS is accurate without requiring corrections for interferences, which is why the accuracy of laser ablation Sr isotope data in the literature is often assessed by reference to TIMS values.

In summary, because of the different ion yields of each analytical method a TIMS analysis will, with current instrumentation, always require less $\mathrm{Sr}$ than either a solution-mode or laser ablation-mode MC-ICPMS analysis to provide a certain level of precision and accuracy on the ${ }^{87} \mathrm{Sr} /{ }^{86} \mathrm{Sr}$ ratio. Alternatively, for a given amount of Sr TIMS will always provide more precise and, more importantly, more accurate Sr isotope data than a laser ablation-mode MC-ICPMS analysis. The amount of material that must be removed from a sample for a TIMS analysis is hence less than that required for a laser ablation analysis. When TIMS is combined with an appropriate microsampling approach (see Section 4.2) the result is an analytical method for Sr isotope measurement that is less destructive than laser ablation.

\subsection{Spatial and compositional resolution of analyses.}

Richards et al (2008) state that micro-sampling by laser ablation allows resolution of 'very small scale migrations which would not be possible with traditional methods'. This is a misleading statement which gives the impression that laser ablation is the only micro sampling method 
available for Sr isotope analysis. Electro-Scientific Industries (New-Wave Research Division), which manufacture the UP213 laser used by Richards et al (2008), also manufacture a Micromill that uses almost identical control software to the UP-series lasers allowing it to be positioned with the same spatial resolution. It is used to excavate material in the form of a fine powder or slurry from a sample using a fine-tipped mill/drill bit (Charlier et al., 2006). The sample powder can then be dissolved and processed through conventional Sr pre-concentration columns and analysed by either of the existing ‘traditional methods' referred to by Richards et al (2008).

Since TaF TIMS requires less Sr for an isotopic analysis of a given precision, combining it with micro-milling can provide similar or better spatial resolution than is possible with laser ablation MC-ICPMS since less sample needs to be removed. More importantly, it combines that greater spatial resolution with much greater levels of precision and accuracy on the measured ${ }^{87} \mathrm{Sr} /{ }^{86} \mathrm{Sr}$ ratio (see sections 4.1 .1 and 4.1.2). This, in turn, allows far greater compositional resolution of $\mathrm{Sr}$ isotope variations within a sample than can ever be achieved by laser ablation MCICPMS.

Probably the most significant advantage of sampling by micro-milling compared to laser ablation that is rarely acknowledged is the greater amount of chemical information that can be acquired from a single sampling point. An aliquot (generally 10\%) of the dissolved sample recovered from a micro-mill spot can be used for a full trace element analysis (eg. Font et al., 2008) before the remaining sample is processed for isotope measurements. Furthermore, concentrations permitting, isotope ratios can be obtained on several elements (eg. Sr and $\mathrm{Pb}$ ).

The most appropriate method for the Sr isotopic analysis of rare and important hominid fossils or samples with low Sr contents, which offers the best chance of guaranteeing precise and accurate Sr isotope data at the first attempt, and with the minimum of sample damage, is not laser ablation but a combination of the micro-milling sampling approach and the long established TaF TIMS analytical method. This is increasingly becoming the approach of choice within the geochemical community for in-situ Sr isotope measurements where precision and accuracy remain paramount and where the limitations of laser ablation MC-ICPMS have been realised over the last 13years.

\section{Conclusions.}

The Richards et al (2008) study presents Sr isotope data for a single Neanderthal tooth that is not accompanied by any Sr isotope data for the local area. This lack of supporting data together with the considerable uncertainty over the accuracy of the Sr isotope data for the Neanderthal tooth 
is such that it is unsafe to consider this data as being the 'first direct evidence for Neanderthal mobility'. The uncertainty with the data in Richards et al. (2008) can be summarised as follows:

- There is no serious attempt to quantify the effect of isobaric interferences save for a rudimentary constant correction based on two non-human standard teeth.

- The concentration of $\mathrm{Sr}$ in the sample is not reported but appears to be quite low, which increases the consequences of matrix related interferences.

- The concentration of $\mathrm{Sr}$ in the sample must vary (Figure 1) and therefore so will the Sr/interference ratio.

- The constant offset correction, as applied to the Neanderthal tooth, cannot yield accurate ${ }^{87} \mathrm{Sr} /{ }^{86} \mathrm{Sr}$ since it does not take into account variable Sr/interference ratios.

Whilst this is not the place to specify exactly how interference corrections should be applied to laser ablation $\mathrm{Sr}$ isotope data we nevertheless strongly recommend that such data always be accompanied by a minimum of key information that allows the accuracy and quality of the final corrected data to be assessed independently; information that was missing from Richards et al (2008). The key information for all reference material and sample analyses that should always be included, in addition to the customary information on mass spectrometer and laser ablation parameters, are listed below:

- Concentration of Sr.

- ${ }^{88} \mathrm{Sr}$ beam intensity.

- Beam intensities for key interference monitor masses.

- Oxide and dimer production rates.

- ${ }^{87} \mathrm{Sr} /{ }^{86} \mathrm{Sr}$ data both with and without interference corrections.

- ${ }^{84} \mathrm{Sr} /{ }^{86} \mathrm{Sr}$ data with interference corrections.

Using an alternative approach to correcting interferences, which tries to take into account variable Sr concentrations and Sr/interference ratios, we suggest that it is equally as valid to argue that the Sr isotope data obtained on the Neanderthal tooth by Richards et al (2008) provides the first direct evidence for Neanderthal immobility. Reanalysing the tooth using a more appropriate analytical method free from isobaric interferences and providing higher quality data might help resolve the migration model although only within the context of a broader study that addresses the local Sr isotope variation.

Laser ablation MC-ICPMS is not necessarily the most rapid method for obtaining Sr isotope ratios. When compared to micro-milling+TIMS it is also a more destructive sampling method and provides worse precision and accuracy on the $\mathrm{Sr}$ isotope composition. The most appropriate 
analytical method for obtaining $\mathrm{Sr}$ isotopes on archaeological artefacts that have low $\mathrm{Sr}$, are small in size and/or are very precious is a combination of micro-milling combined with TIMS.

\section{References}

Bentley, R.A. 2006. Strontium isotopes from the Earth to the archaeological skeleton: a review. Journal of Archaeological Method and Theory 13: 135-187.

Bentley, R.A., Price, T.D., and Stephan, E. 2004. Determining the 'local' ${ }^{87} \mathrm{Sr} /{ }^{86} \mathrm{Sr}$ range for archaeological skeletons: a case study from Neolithic Europe. Journal of Archaeological Science. 31, 365-375.

Birk, J.L. 1986. Precision K-Rb-Sr isotopic analysis: application to Rb-Sr chronology. Chemical Geology, 56, 73-83.

Charlier, B.L.A., Ginibre, C., Morgan, D., Nowell, G.M., Pearson, D.G., Davidson, J.P and Ottley, C.J. 2006. Methods for the microsampling and high-precision analysis of strontium and rubidium isotopes at single crystal scale for petrological and geochronological applications. Chemical Geology, 232, 114-133.

Copeland, S.R., Sponheimer, M, le Roux, P.J., Grimes, V., Lee-Thorp, J.A., Darryl J. de Ruiter, D.J. and Richards, M.P. 2008. Strontium isotope ratios $\left({ }^{87} \mathrm{Sr} /{ }^{86} \mathrm{Sr}\right)$ of tooth enamel: a comparison of solution and laser ablation multicollector inductively coupled plasma mass spectrometry methods. Rapid Communications in mass Spectrometry. 22, 3187-3194

Font, L., Davidson, J.P., Pearson, D.G., Nowell, G.M., Jerram, D.A., and Ottley, C.J. 2008. Sr and $\mathrm{Pb}$ IsotopeMicro-analysis of Plagioclase Crystals from Skye Lavas: an Insight into Open-system Processes in a Flood Basalt Provinc.Journal opf Petrology, 49 No.8, 1449-1471

Hostwood, M.S.A., Evans, J., and Montgomery, J. 2008. Determination of Sr isotopes in calcium phosphates using laser ablation inductively coupled plasma mass spectrometry and their application to archaeological tooth enamel. Geochim. et Cosmochim acta. 72, 5659-5674

J. H. Batey, T. Prohaska, M.S.A. Horstwood, G.M. Nowell, H. Goenaga-Infante \& G. C. Eiden 2005. Mass Spectrometers. In ICP Mass Spectrometry Handbook. S. Nelms Blackwell. 26-116.

Montgomery, J., Evans, J. A., and Neighbour, T. 2003. Sr isotope evidence for population movement within the Hebridean Norse community of NW Scotland. Journal of the Geological Society 160: 649-653.

Richards, M., Harvati, K., Grimes, V., Colin Smith, C., Smith, T., Jean-Jacques Hublin, J.J., Karkanas, P., and Panagopoulou, E. 2008. Strontium isotope evidence of Neanderthal mobility at the site of Lakonis, Greece using laser ablation PIMMS. Journal of Archaeological Science 35, 1251-1256.

Simonetti, A., Buzon, M.R., and Creaser, R.A. 2008. In-situ elemental and Sr isotope investigation of human tooth enamel by laser ablation- (MC)-ICP-MS: Successes and pitfalls. Archaeometry 50, 371-385. 
Waight, T., Baker, J., Peate, D. 2002. Sr isotope ratio measurements by double-focusing MCICPMS: techniques, observations and pitfalls International Journal of Mass Spectrometry 221:229244

Vroon, P.Z., van der Wagt, B., Koornneef, J.M., and Davies, G. 2008. Problems in obtaining precise and accurate $\mathrm{Sr}$ isotope analysis from geological materials using laser ablation MC-ICPMS. Analytical and Bioanalytical Chemistry. 390, 465-476. 